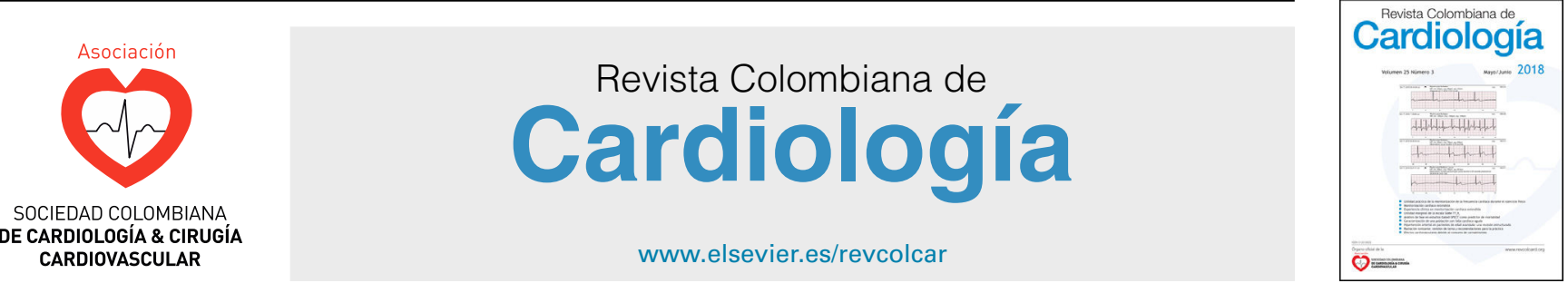

CARDIOLOGÍA DEL ADULTO-ORIGINAL

\title{
Acuerdo inter- e intraobservador de la cuantificación del ángulo QRS-T espacial calculado por el método de transformación visual. Estudio piloto
}

\author{
Juan Morales ${ }^{\mathrm{a}, *}$, Álvaro Herrera ${ }^{\mathrm{b}}$, Luis M. Herrera ${ }^{\mathrm{c}}$, Juan D. Lemos ${ }^{\mathrm{c}}$, \\ María E. Casanova ${ }^{\mathrm{b}, \mathrm{c}}$ y Marisol Badiel ${ }^{\mathrm{a}, \mathrm{c}}$
}

\author{
a Universidad del Valle, Cali, Colombia \\ b DIME, Clínica neurocardiovascular, Cali, Colombia \\ c Universidad Libre, Cali, Colombia
}

Recibido el 2 de junio de 2018; aceptado el 7 de noviembre de 2018

Disponible en Internet el 6 de junio de 2019

\section{PALABRAS CLAVE \\ Ángulo QRS-T; \\ Reproducibilidad de resultados; \\ Electrocardiografía; \\ Vectorcardiografía}

\begin{abstract}
Resumen
Introducción: El ángulo QRS-T espacial es la diferencia entre el vector del QRS y la T. Se conoce el valor diagnóstico y pronóstico de este marcador en enfermedades cardíacas. Es prioritario determinar si estas mediciones son reproducibles con confiabilidad en nuestro medio.

Métodos: En 30 adultos se obtuvieron electrocardiogramas, independientemente del diagnóstico. Las mediciones las realizaron dos estudiantes de Medicina de quinto año, un médico interno y un cardiólogo, posterior a un reentrenamiento sobre la medición del ángulo por el método de transformación visual.

Con identificación cegada, ingresaron en un aplicativo web las mediciones del QRS y la T. El ángulo fue estimado a partir de la plantilla de Cortez y colaboradores. Sobre el $20 \%$ de las lecturas se estimó un acuerdo intraobservador y en el $100 \%$ de las lecturas un acuerdo interobservador. Los puntos de corte para estimar el acuerdo Kappa fueron $<105^{\circ}$ (normal), $105^{\circ}$ a $135^{\circ}$ (limítrofe) $y>135^{\circ}$ (anormal).

Resultados: Se excluyó un ECG por calidad deficiente. El rango del ángulo estuvo entre $72.9^{\circ}$ y $176.2^{\circ}$. El acuerdo interobservador entre los 4 evaluadores fue kappa $=0.786$ (IC95\% 0.728 $0.823, \mathrm{p}<0.0001$ ) y el intraobservador kappa $=1.0$ ( $\mathrm{p} 0.007$ ).

Conclusión: Es el primer estudio sobre el ángulo QRS-T espacial realizado en Colombia. Se logró un acuerdo inter- e intraobservador adecuado en la medición del ángulo por el método de transformación visual, en observadores con diferente experticia, con un kappa mayor de 0,75. Este resultado apoya la reproducibilidad de esta medición en estudios posteriores en Colombia. (c) 2019 Sociedad Colombiana de Cardiología y Cirugía Cardiovascular. Publicado por Elsevier España, S.L.U. Este es un artículo Open Access bajo la licencia CC BY-NC-ND (http:// creativecommons.org/licenses/by-nc-nd/4.0/).
\end{abstract}

\footnotetext{
* Autor para correspondencia.

Correo electrónico: juan.morales@correounivalle.edu.co (J. Morales).
} 


\section{KEYWORDS}

QRS-T Angle;

Reproducibility of results; Electrocardiography; Vectorcardiography
Inter- and intra-observer agreement in the quantification of the spatial QRS-T angle calculated by visual transformation. A pilot study

\begin{abstract}
Introduction: The spatial QRS-T angle is the difference between the QRS vector and T vector. The diagnostic and prognostic value of this marker in heart disease is well-known. It is essential to determine whether these measurements are reproducible and reliable in Colombia.

Methods: Electrocardiograms (ECG) were obtained from 30 adults, regardless of the diagnosis. The measurements were made by two fifth-year medical students, a houseman/ intern, and a cardiologist. They were all re-trained on the measurement of the angle using the visual transformation method.

With blinded identification, they entered the QRS and T measurements into a web application. The angle was estimated from the template of Cortez et al. About $20 \%$ of the readers showed an intra-observer agreement and an inter-observer agreement in $100 \%$. The cut-off points to estimate the agreement Kappa were $<105^{\circ}$ (normal), $105^{\circ}$ a $135^{\circ}$ (borderline), and $>135^{\circ}$ (abnormal).

Results: One ECG was excluded due to poor quality. The range of the angle was between $72.9^{\circ}$ and $176.2^{\circ}$. The inter-observer agreement between the 4 evaluators gave a kappa $=0.786$ (95\% $\mathrm{Cl}$; 0.728-0.823, $P<.0001)$, and the intra-observer agreement a kappa $=1.0(P=.007)$.

Conclusion: This the first study on the spatial QRS-T angle performed in Colombia. A good interand intra-observer agreement was achieved in the measurement of the angle by the visual transformation method by observers with different levels of expertise, with a Kappa greater than 0.75 . These results support the reproducibility of this measurement in subsequent studies in Colombia.

(c) 2019 Sociedad Colombiana de Cardiología y Cirugía Cardiovascular. Published by Elsevier España, S.L.U. This is an open access article under the CC BY-NC-ND license (http:// creativecommons.org/licenses/by-nc-nd/4.0/).
\end{abstract}

\section{Introducción}

El electrocardiograma de superficie (ECG) es un método diagnóstico de gran utilidad y aún vigente, que permite conocer la actividad eléctrica del corazón en función del tiempo. Algunas enfermedades que presenta el ECG han cumplido la función de marcadores diagnósticos para pronóstico de algunas patologías cardiovasculares, entre ellas la hipertrofia ventricular ${ }^{1}$, la muerte súbita cardíaca ${ }^{2,3}$, la displasia arritmogénica del ventrículo derecho ${ }^{4}$, entre otras.

Desde inicios de 2000, un nuevo marcador electrocardiográfico ha llamado la atención de algunos investigadores ${ }^{5}$, reconociendo al complejo QRS como la representación de la despolarización ventricular y la onda $T$ como la representación de la repolarización ventricular, así como los vectores que son producto de la actividad eléctrica de los cardiomiocitos en estos dos procesos eléctricos. Se comprende que ciertas alteraciones cardíacas conllevan cambios en la despolarización y repolarización ventricular representadas en el ECG de superficie.

De esta misma forma, el ángulo QRS-T resultante de la diferencia entre el vector de despolarización y repolarización cardíaca, representado en grados en el triángulo de Einthoven, indica qué tan alejado (ángulo QRS-T abierto) o qué tan cercano (ángulo QRS-T estrecho) se encuentra el vector de despolarización, respecto a la repolarización.

El ángulo QRS-T se puede calcular de diferentes formas; las dos más importantes son: en los planos frontal y transversal y en el espacial ${ }^{6}$. Los planos frontal y transversal incluyen las 12 derivaciones clásicas del ECG. Es la forma más sencilla de cálculo, pero poco precisa. Por otro lado, el cálculo del ángulo QRS-T espacial da una mayor precisión, pero requiere de métodos complejos para su medición ${ }^{7}$.

Estudios recientes han llamado la atención del valor del ángulo QRS-T para identificar alteraciones cardíacas a partir de la aproximación espacial ${ }^{8}$ y se han descrito y probado métodos matemáticos para estimar la cuantificación del ángulo QRS-T de manera más práctica9 .

Este estudio pone en práctica una forma sencilla y altamente precisa de hallar el ángulo QRS-T espacial. Por ello el objetivo de este estudio piloto es determinar el acuerdo inter- e intraobservador de la cuantificación del ángulo QRS-T espacial calculado por el método de transformación visual. La hipótesis es que en personal médico con diferente experticia y con entrenamiento adecuado en la lectura del electrocardiograma convencional, puede reproducirse los resultados de dicha medición. Si el método de medición es reproducible se puede continuar el estudio en pacientes específicos y determinar pronóstico en ellos, conforme se está reconociendo su utilidad en otros escenarios.

\section{Planteamiento del problema}

El cálculo de los vectores del QRS y la onda T han sido campo de investigación desde antes de los años 30 . Métodos clásicos por los cuales se calculaban estas magnitudes se encuentran en desuso actualmente, pero la importancia clínica de reconocerlos perdura en el tiempo ${ }^{10}$. 
Debido a lo anterior, diversos investigadores han planteado métodos alternativos para el cálculo de estos vectores y sobre todo, de la diferencia entre el vector del QRS y la onda T. Entre ellos se encuentran el método de inversión de Dower, el de regresión de Kors y la fórmula coseno inverso de Rautaharju. Aunque estos métodos tienen una sensibilidad y especificidad más alta que el método del plano frontal, no deja de ser dispendiosa y poco práctica la manera de ejecutarlos ${ }^{11}$.

Por esto, se vio la necesidad de aplicar una forma más sencilla y reproducible. En el año 2014, Cortez y colaboradores encontraron una forma que llenaba estos requisitos. El método de transformación visual logra tener una sensibilidad y especificidad más altas que sus antecesores y se calcula tan fácilmente como el método del plano frontal ${ }^{9}$. A pesar de este aporte en investigación traslacional, hay pocos estudios que apliquen este método y contribuyan a su validación. Por consiguiente, se desconocen los alcances que pudiese tener el cálculo del ángulo QRS-T espacial por el método de transformación visual. Es necesaria la validación de esta herramienta, la cual podría permitir la obtención de uno de los predictores electrocardiográficos de mayor impacto.

\section{Métodos}

Estudio exploratorio, fase piloto de pruebas diagnósticas ${ }^{12}$. De manera prospectiva, entre noviembre y diciembre de 2017 y previa firma de consentimiento informado, se recolectaron 30 trazados electrocardiográficos en igual número de sujetos mayores de 18 años, con cualquier diagnóstico hospitalizados en una IPS nivel 3 de la ciudad de Cali, Colombia seleccionados de forma aleatoria. Se excluyeron trazados con calidad deficiente que impidiera su lectura. Este estudio fue aprobado por los comités de ética de la Universidad Libre y la IPS hospitalaria.

\section{Procedimiento}

Trazado del electrocardiograma. Para la toma de los trazados se construyó una lista de chequeo del procedimiento, donde se especifican los aspectos técnicos para la toma del electrocardiograma estándar. Se siguieron las recomendaciones del American College of Cardiology ${ }^{13}$.

Medición del QRS-T. Se midió en milímetros tanto el complejo QRS como la onda T en las derivaciones I, II y V1 a V6, considerando toda deflexión por encima de la línea de base (segmento TP) como positiva y toda deflexión por debajo de la línea de base como negativa (fig. 1). Posteriormente, los valores obtenidos en milímetros se introdujeron en la plantilla de Excel $^{\circledR}$, en las celdas que corresponden a cada derivación. Para las deflexiones que fuesen perfectamente isobifásicas se consideró el valor de cero milímetros. Los valores con decimales se permitieron, utilizando la coma para separar el número entero de sus decimales (fig. 2).

Previo a la toma de cada trazado se le explicó el procedimiento a cada uno de los pacientes, así como el diligenciamiento del consentimiento informado.

En una plataforma de captura basada en web, se introdujeron las mediciones. Posteriormente, la base de datos se transformó en una plantilla de Excel $^{\circledR}$ para realizar los cálculos del ángulo QRS-T.

Para el ángulo QRS-T espacial en X, Y y Z la plantilla realiza la sumatoria de los valores introducidos multiplicándolos por los coeficientes obtenidos por el método de regresión y cuasiortogonal de Kors (tabla 1).

De acuerdo con lo anterior, con los valores del ángulo QRS y T espaciales en los 3 planos se obtiene un valor que representa el ángulo en los 3 planos para el QRS y la T mediante la siguiente fórmula:

Para el QRS = RAIZ $((\mathrm{B} 6) \hat{2}+(\mathrm{B} 7) \hat{2}+(\mathrm{ABS}(\mathrm{B} 8)) \hat{2})$

Para la T = RAIZ ((ABS(C6)) $\hat{2}+(C 7) \hat{2}+(A B S(C 8)) \hat{2})$

Una vez con los ángulos calculados, se multiplican $y$ suman los ángulos del QRS y la T en cada plano (X, Y y Z) y se dividen por el producto del ángulo QRS y $\mathrm{T}$ espaciales.

$=A C O S\left(\left(\left(B 6^{*} \mathrm{C} 6\right)+\left(B 7^{*} \mathrm{C} 7\right)+\left(B 8^{*} \mathrm{C} 8\right)\right) /\left(\mathrm{E} 10^{*} \mathrm{E} 11\right)\right)$.

Por último, se obtiene el valor que representa la diferencia entre el ángulo espacial del QRS y de la T en las 8 derivaciones evaluadas.

$=\left(D 13^{*} 180\right) /$ La diferencia de los ángulos espaciales del QRS y la T.

Todos los procesos previamente nombrados los realiza la plantilla de forma automática, en tiempo real, y el usuario solo debe introducir los valores de las deflexiones en milímetros. Para más detalles sobre la racionalidad del estimado se recomienda el artículo de Cortez et al. ${ }^{9}$

\section{Preparación del personal médico}

En la medición del ángulo QRS-T espacial, los 4 observadores ( 2 estudiantes de Medicina de quinto año, 1 médico interno y 1 cardiólogo) fueron cegados respecto a la procedencia del electrocardiograma y los datos clínicos o de otra índole perteneciente al sujeto de quien se obtuvo el trazado. El reentrenamiento en lectura del electrocardiograma fue ofrecido por el cardiólogo (AH) y por el médico interno (JM) conforme la recomendación del estándar internacional ${ }^{13}$.

\section{Análisis estadístico}

El tamaño de muestra fue a conveniencia para un estudio exploratorio piloto fase $1^{12}$. Una vez obtenidos los datos de las mediciones, se realizó un análisis exploratorio y descriptivo de cada variable. Se estimaron los promedios y la desviación estándar en las variables continuas con distribución normal o mediana y rangos intercuartílicos en las que presentan distribución no normal.

Para estimar el coeficiente Kappa, se construyeron tablas de contingencia. Se consideró que existe un buen acuerdo interobservador si el índice de Kappa es mayor o igual al 70\% en cada derivación. Los pesos ponderados para cada celda fueron iguales ${ }^{14}$.

Se establecieron como puntos de corte para convertir las variables continuas en categóricas en los parámetros de normalidad. Es así que para el ángulo QRS-T espacial los intervalos menores a $105^{\circ}$ fueron considerados como normales, de $105^{\circ}$ a $135^{\circ}$ como limítrofes y mayores a $135^{\circ}$ anormales. Esta clasificación se usa frecuentemente en estudios previos ${ }^{8}$.

Todos los análisis se realizaron en STATA ${ }^{\circledR}$ v12.0. 


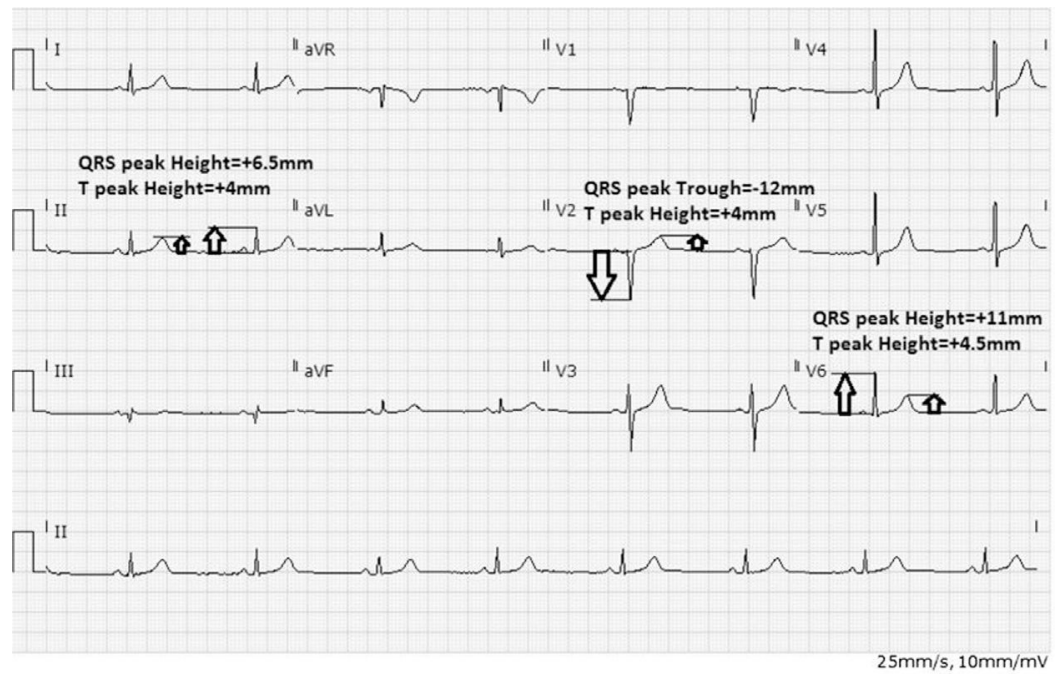

Figura 1 Criterios de medición del QRS-T en el electrocardiograma.

Medición de la amplitud del complejo QRS y la onda T en milímetros, a partir del segmento TP. Tomado con permiso del Dr. Daniel Cortez.

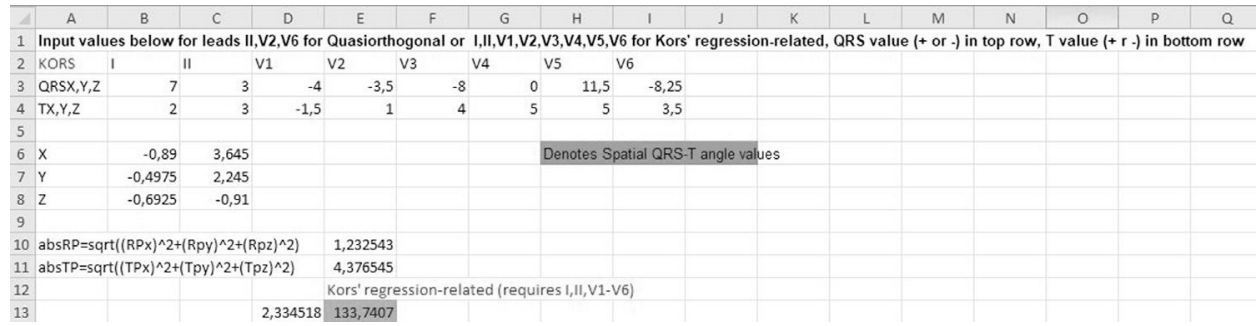

Figura 2 Plantilla de Excel $^{\circledR}$.

Los valores obtenidos de la amplitud del QRS y la onda T se introducen en la celda correspondiente, antecedidos del signo menos si es una deflexión negativa. Si no son valores entero se permite introducir decimales, separados por una coma.

Tabla 1 Coeficientes $\mathrm{X}, \mathrm{Y}, \mathrm{Z}$ en las 8 derivaciones evaluadas

\begin{tabular}{|c|c|c|c|c|c|c|c|c|c|}
\hline & & I & II & VI & V2 & V3 & V4 & V5 & V \\
\hline \multirow[t]{3}{*}{ Kors } & $x$ & 0,38 & $-0-07$ & $-0,13$ & 0,05 & $-0,01$ & 0,14 & 0,06 & 0,54 \\
\hline & $Y$ & $-0,07$ & 0,93 & 0,06 & $-0,02$ & $-0,05$ & 0,06 & $-0,17$ & 0,13 \\
\hline & Z & 0,11 & $-0,23$ & $-0,43$ & $-0,06$ & $-0,14$ & $-0,2$ & $-0,11$ & 0,31 \\
\hline
\end{tabular}

\section{Resultados}

Se obtuvieron en total 30 trazados electrocardiográficos; se excluyó un trazado en todos los análisis puesto que uno de los evaluadores observó una dificultad técnica. El rango para el cálculo del ángulo QRS-T espacial entre todas las mediciones de los observadores fue desde $72,9^{\circ}$ a $176,2^{\circ}$.

En la tabla 2 se presentan los demás parámetros de medición. El acuerdo interobservador entre los 4 evaluadores fue kappa = 0,786 (IC95\% 0,728-0,823, p<0,0001) (tabla 3). Adicionalmente, en la tabla 4 se presentan todos los acuerdos intraobservador los cuales tuvieron un kappa $=1,0$ ( $p$ 0,007) para cada observador.

\section{Discusión}

Este el primer estudio sobre el ángulo QRS-T espacial realizado en Colombia. En este piloto se obtiene un acuerdo inter- e intraobservador en la medición del ángulo por el método de transformación visual, en observadores con diferente experticia, con la estimación de un coeficiente de kappa mayor a 0,75. Los hallazgos encontrados indican que la medición del ángulo QRS-T espacial por el método de transformación visual es factible y reproducible, incluso en observadores con diferentes niveles de experticia. El ángulo QRS-T es considerado un marcador electrocardiográfico relevante ${ }^{15,16}$, sin embargo hasta el momento no se han 
Tabla 2 Características generales de las mediciones por observador

\begin{tabular}{|c|c|c|c|c|}
\hline Observación & Observador1 & Observador2 & Observador3 & Observador4 \\
\hline \multicolumn{5}{|l|}{ Frecuencia cardíaca } \\
\hline Promedio $\pm \mathrm{DE}$ & $64,6 \pm 10,7$ & $77,5 \pm 18,8$ & $73,4 \pm 12,3$ & $76,7 \pm 16,7$ \\
\hline Rango intercuartílico & $62-81$ & $68-88$ & $66-85$ & $66-87$ \\
\hline \multicolumn{5}{|l|}{ Complejo QRS } \\
\hline Promedio $\pm \mathrm{DE}$ & $82,4 \pm 42,2$ & $91,1 \pm 49,6$ & $85,1 \pm 48,9$ & $93,1 \pm 48,1$ \\
\hline Rango intercuartílico & $45,8-122,3$ & $41,4-136,7$ & $37,7-128,5$ & $46,1-139,6$ \\
\hline \multicolumn{5}{|l|}{ Onda $T$} \\
\hline Promedio $\pm \mathrm{DE}$ & $75 \pm 37$ & $96,5 \pm 46,8$ & $87 \pm 46,1$ & $94,6 \pm 46,4$ \\
\hline Rango intercuartílico & $42,1-109,5$ & $60,5-139,1$ & $53,3-124,2$ & $59,3-137$ \\
\hline
\end{tabular}

Tabla 3 Acuerdo interobservador estimado de kappa con IC95\%, por parejas de la medición en seis electrocardiogramas del ángulo QRS-T espacial por método de transformación visual

\begin{tabular}{|c|c|c|c|c|}
\hline Ángulo QRS-T espacial & Observador1 & Observador2 & Observador3 & Observador4 \\
\hline Observador1 & & & & $0,88(0,73-1,00)$ \\
\hline Observador2 & $0,76(0.55-0,98)$ & & & $0,88(0,73-1,00)$ \\
\hline Observador3 & $0,64(0,38-0,90)$ & $0,76(0,55-0,98)$ & & $0,88(0,72-1,00)$ \\
\hline Observador4 & $0,88(0,73-1,00)$ & $0,88(0,73-1,00)$ & $0,88(0,72-1,00)$ & \\
\hline
\end{tabular}

Tabla 4 Acuerdo intraobservador estimado de kappa con IC95\% de la medición en seis electrocardiogramas del ángulo QRS-T espacial por método de transformación visual

\begin{tabular}{|c|c|c|c|c|}
\hline Ángulo QRS-T espacial & Observador1 & Observador2 & Observador3 & Observador4 \\
\hline Observador1 & $1,0(1,0-1,0)$ & & & \\
\hline Observador2 & & $1,0(1,0-1,0)$ & & \\
\hline Observador3 & & & $1,0(1,0-1,0)$ & \\
\hline Observador4 & & & & $1,0(1,0-1,0)$ \\
\hline
\end{tabular}

realizado estudios en Colombia. En la mayoría de estudios descritos en la literatura estas mediciones se hacen de forma automática a través de programas de computadora ${ }^{17,18}$ o con un observador único ${ }^{19}$. En nuestro medio, dichas mediciones masivas son poco viables debido a los limitados recursos tecnológicos y a las escasas bases de registros de electrocardiogramas digitalizados. Pese a ello, lograr un acuerdo inter e intraobservador adecuado en la medición manual del ángulo QRS-T espacial hace más real la posibilidad de hacer estas mediciones al pie de la cama del paciente.

Este estudio es el primero en Colombia que involucra al ángulo QRS-T espacial. Por lo tanto, se convierte en un referente importante para continuar explorando los alcances de este marcador. En trabajos anteriores ha sido posible identificar su relación diagnóstica y pronóstica en enfermedades

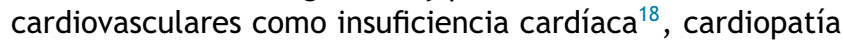
isquémica ${ }^{20}$ y muerte de origen cardíaco $^{8,21}$. Sin embargo, en población latinoamericana estos datos son muy escasos y mucho más aún, en población colombiana.

Para responder la pregunta de investigación que se planteó se establecieron estrategias para minimizar al máximo los sesgos, como fue un entrenamiento previo a la medición y garantizar el cegamiento de los trazados electrocardiográficos entre los observadores. Adicionalmente, el análisis estadístico estuvo a cargo de una persona diferente a los observadores (MB).

\section{Conclusiones}

Es el primer estudio sobre el ángulo QRS-T espacial hecho en Colombia. Se logró un acuerdo inter- e intraobservador adecuado en la medición del ángulo por el método de transformación visual, en observadores con diferente experticia, con la estimación de un índice de kappa mayor a 0,75. Esto apoyará la reproducibilidad de esta medición en estudios posteriores en Colombia.

\section{Financiación}

Convocatoria interna Universidad Libre, seccional Cali, Colombia (ID del proyecto CIFSC-2017-OCT-04-008).

\section{Conflicto de intereses}

Ninguno. 


\section{Agradecimientos}

Al Dr. Daniel Cortez (Hershey, PA, USA), por sus valiosos aportes en el conocimiento de la medición.

En la Universidad Libre - Campus Cali: Al Dr. Iván Leonardo Cepeda, director de Investigaciones de la Facultad de Ciencias de la Salud, quien garantizó la logística del trabajo; al Dr. Eider Moreno como coordinador del estudio y al señor Wolfan Núñez por el desarrollo de la plataforma web de captura y base de datos.

\section{Bibliografía}

1. Casale PN, Devereux RB, Kligfield P, Eisenberg RR, Miller DH, Chaudhary BS, et al. Electrocardiographic detection of left ventricular hypertrophy: development and prospective validation of improved criteria. J Am Coll Cardiol. 1985;6:572-80.

2. Yamazaki T, Froelicher VF, Myers J, Chun S, Wang P. Spatial QRS$T$ angle predicts cardiac death in a clinical population. Heart Rhythm. 2005;2:73-8.

3. Antzelevitch C, Brugada P, Borggrefe M, Brugada J, Brugada $\mathrm{R}$, Corrado $\mathrm{D}$, et al. Brugada syndrome: report of the second consensus conference. Circulation. 2005;111:659-70.

4. Nasir K, Bomma C, Tandri H, Roguin A, Dalal D, Prakasa K, et al. Electrocardiographic features of arrhythmogenic right ventricular dysplasia/cardiomyopathy according to disease severity. Circulation. 2004;110:1527-34.

5. Kardys I, Kors JA, van der Meer IM, Hofman A, van der Kuip DA, Witteman JC. Spatial QRS-T angle predicts cardiac death in a general population. Eur Heart J. 2003;24:1357-64.

6. Oehler A, Feldman T, Henrikson CA, Tereshchenko LG. QRS$T$ angle: a review. Annals of Noninvasive Electrocardiology. 2014;19:534-42.

7. Rautaharju PM, Prineas RJ, Zhang Z-M. A simple procedure for estimation of the spatial QRS/T angle from the standard 12-lead electrocardiogram. J Electrocardiol. 2007;40:300-4.

8. Zhang X, Zhu Q, Zhu L, Jiang H, Xie J, Huang W, et al. Spatial/frontal QRS-T angle predicts all-cause mortality and cardiac mortality: A meta-analysis. PloS one. 2015;10:e0136174.

9. Cortez D, Sharma N, Devers C, Devers E, Schlegel TT. Visual transform applications for estimating the spatial QRS-T angle from the conventional 12-lead ECG: Kors is still most Frank. J Electrocardiol. 2014;47:12-9.

10. Frank E. An accurate, clinically practical system for spatial vectorcardiography. circulation. 1956;13:737-49.
11. Kors J, Van Herpen G, Sittig A, Van Bemmel J. Reconstruction of the Frank vectorcardiogram from standard electrocardiographic leads: diagnostic comparison of different methods. Eur Heart J. 1990; 11:1083-92.

12. Pepe MS. The statistical evaluation of medical tests for classification and prediction: Medicine. 2003.

13. Kligfield P, Gettes LS, Bailey JJ, Childers R, Deal BJ, Hancock EW, et al. Recommendations for the standardization and interpretation of the electrocardiogram: part I: the electrocardiogram and its technology: a Scientific Statement from the American Heart Association Electrocardiography and Arrhythmias Committee Council on Clinical Cardiology; the American College of Cardiology Foundation; and the Heart Rhythm Society. Endorsed by the International Society for Computerized Electrocardiology. Heart Rhythm. 2007;4: 394-412.

14. Landis JR, Koch GG. An application of hierarchical kappa-type statistics in the assessment of majority agreement among multiple observers. Biometrics. 1977:363-74.

15. Hnatkova K, Seegers J, Barthel P, Novotny T, Smetana P, Zabel $M$, et al. Clinical value of different QRS-T angle expressions. EP Europace. 2017.

16. Birnbaum Y, Nikus K. Electrocardiographic risk stratification of asymptomatic population without cardiovascular disease Should we add the QRS-T angle? Elsevier. 2017.

17. Cortez D, Schlegel TT, Ackerman MJ, Bos JM. ECG-derived spatial QRS-T angle is strongly associated with hypertrophic cardiomyopathy. Journal of electrocardiology. 2017;50:195-202.

18. Rautaharju PM, Kooperberg C, Larson JC, LaCroix A. Electrocardiographic predictors of incident congestive heart failure and all-cause mortality in postmenopausal women. Circulation. 2006;113:481-9.

19. Selvaraj S, Ilkhanoff L, Burke MA, Freed BH, Lang RM, Martinez $\mathrm{EE}$, et al. Association of the frontal QRS-T angle with adverse cardiac remodeling, impaired left and right ventricular function, and worse outcomes in heart failure with preserved ejection fraction. J Am Soc Echocardiography. 2014;27:74-82, e2.

20. Zhang Z-m, Rautaharju PM, Prineas RJ, Tereshchenko L, Soliman EZ. Electrocardiographic QRS-T angle and the risk of incident silent myocardial infarction in the Atherosclerosis Risk in Communities study. J Electrocardiol. 2017.

21. Laukkanen JA, Di Angelantonio E, Khan H, Kurl S, Ronkainen K, Rautaharju P. T-wave inversion QRS duration, and QRS/T angle as electrocardiographic predictors of the risk for sudden cardiac death. Am J Cardiol. 2014;113:1178-83. 